\title{
Unabhängigkeit der Justiz und gesellschaftliche Veränderung
}

Der im folgenden abgedruckte Text ist die (von Hans-Ernst Böttcher angefertigte) deutsche Übersetzung der nachträglich erstellten schriftlichen Fassung eines Diskussionsbeitrages vom 14 . Kongreß der französischen Richtergewerkschaft »syndicat de la magistrature (November 1981), dem ersten seit der Regierungsübernabme durch Mitterand und die Linksunion im Mai/Juni 1981.

Salvatore Senese, der für die italienische Richtergewerkschaft "magistratura democratica " als Gast an dem Kongreß teilnabm, griff in die Debatte ein, als es um die Definition der Unabhängigkeit der Justiz im Verbältnis zu der neuen, der linken Minderbeit in der Justiz näberstebenden Regierung ging.

Unabhängigkeit nach wie vor als strategischer Wert, Unabbängigkeit auch gegenüber der neuen Regierung - diese These hatte vor allem Etienne Bloch (Richter am Appellationsgericht in Versailles), einer der "großen Alten * des "syndicat de la magistrature", aufgestellt. Als die These in der Diskussion von nicht wenigen Kolleginnen und Kollegen angezweifelt wurde und der Kampf für die Unabbängigkeit der Justiz allenfalls zu einem zweitrangigen Ziel erklärt zu werden drobte, griff Senese ein und entwarf aus dem Stegreif - in französischer Sprache - die folgende Skizze, die ich, ungeachtet einiger diskussions- und (im guten Sinne des Wortes) fragwürdiger Details, für den im westeuropäischen Raum bisher schlüssigsten Beitrag zu der auch in der Bundesrepublik gefübrten Diskussion um Notwendigkeit, Voraussetzungen, Möglichkeiten, Grenzen, Gefabren und Funktion einer unabhängigen Justiz balte. Erstaunlicherweise ist der Text bis 1983 nicht und dann nur stark gekürzt in der Zeitschrift "justice " des »syndicat de la magistrature ", für die er in schriftlicher Fassung gedacht war, abgedruckt worden. Die Fußnoten im Text sind vom Übersetzer. [Hans-Ernst Böttcher]

Für einen Richterkongreß zum Thema »justice et changement « war es kaum zu vermeiden, daß im Verlaufe der Debatten das Thema »Unabhängigkeit der Justiz « angesprochen würde; ebenso war klar, daß es darüber zu heftigem Streit kommen mußte, denn die Unabhängigkeit der Dritten Gewalt hat häufig denen als Schlagwort gedient, die gegen jede Veränderung sind. Jedenfalls haben einige Kollegen sich heftigst gegen die Forderung nach der Unabhängigkeit der Justiz gewandt und es vorgezogen, die Forderung nach der Freibeit (Unabhängigkeit) des Richters, Pluralismus im Innern der Richterschaft und nach einer Institution Justiz, die gesellschaftlich etwas bewegt, zu erheben. Über diese Ziele scheint, im Gegensatz zur Unabhängigkeit der Justiz, Einmütigkeit im »syndicat de la magistrature« (»s. m.«) zu bestehen. Von daher bietet es sich an, diese Ziele näher zu untersuchen.

Die Freiheit (Unabhängigkeit) des Richters erscheint von vornherein als Hauptziel, denn sie ist Voraussetzung sowohl für den Pluralismus als auch dafür, daß die Justiz zu einem Faktor gesellschaftlichen Wandels werden kann: In der Tat können sich 
nur dort, wo alle Richter gleichermaßen frei und unabhängig sind, unterschiedlic Strömungen und Meinungen vollständig entfalten und so einen rechtswissenscha: lichen Diskurs gewährleisten, der diesen Namen verdient und der auch zu Bewegu। auf dem Markt der in Lehre und Rechtsprechung herrschenden Meinungen führı kann.

Unabhängigkeit des Richters, also gut! Aber Unabhängigkeit wem gegenüb، Unabhängigkeit wovon, und vor allem: wie soll sie realisiert werden?

Es handelt sich offensichtlich um die Unabhängigkeit des Richters gegenüber de Staatsapparat, seinen Geboten und Pressionen; es handelt sich um die Freiheit, ei direkte Beziehung mit dem Gesetz und dem Lebenssachverhalt, über den Rec gesprochen werden soll, herzustellen, ohne daß sich eine Struktur der Exekuti zwischen die Anwendung des Gesetzes und den Richter schiebt. Diese Freiheit kar sich nicht verwirklichen ohne institutionelle Maßnahmen, die die Existenz solch Zwischenebenen verhindern; man muß dazu die Justiz in einer Weise organisiere daß jeder einzelne Richter gegen die Pressionen des Staatsapparates gefeit ist u1 ihnen entgehen kann.

Nun, die meisten dieser Maßnahmen hätten darauf zu zielen, daß die Riegel, die unseren Ländern die Richtergesetze und die Gerichtsverfassungen einer wirklich Unabhängigkeit vorgeschoben haben, beseitigt werden; das Karrieresystem und $d$ Hierarchie, die Befugnisse der Gerichtspräsidenten, was die Geschäftsverteilung ur die Verteilung der Richter auf die einzelnen Posten angeht ${ }^{t}$, das Beurteilungsw sen ... kurz: all das, wogegen das "s. m. " ankämpft, seitdem ich es kenne. Zu diest "negativen« Forderungen fügt »s. m." heute eine positive hinzu: die Einrichtuı eines obersten Richterrats, der eine effektive und plurale Mitbestimmung aller Ricl ter bei der Justizverwaltung sicherstellt; dieser Richterrat soll zugleich ein Verbil dungsglied darstellen nicht nur zur Regierung, sondern zur Ebene der Politik in gesamt.

Nun - die Summe dieser Forderungen ist nichts anderes als die Forderung na einem System, das die Unabhängigkeit der Richter institutionell garantiert. Tatsäc] lich ist diese Unabhängigkeit, die in jedem liberalen politischen System als essenti betrachtet wird, in der Realität Kontinentaleuropas weithin denaturiert durch eil gesetzlich fixierte hierarchische Struktur der Richterschaft; sie geht auf das napole nische Modell zurück. Dieses Modell hat die richterliche Profession derjenigen d Militärs angeglichen, ist doch das hierarchische Modell weit eher geeignet zur Übe mittlung eines Befehls als zur Entfaltung eines Gedankens.

Mit diesem Kunstgriff, der darin besteht, den Angehörigen der Justiz institutionel Strukturen vorzugeben, die dem Kern ihrer beruflichen Tätigkeit diametral entg gengesetzt sind, ist die Unabhängigkeit der Justiz - mehr oder weniger, je nach de politischen Umständen - eine Fassade geblieben, etwas, wovon im Gesetz die Rec ist, das aber tatsächlich wenig realisiert ist.

Die institutionellen Hindernisse für die Unabhängigkeit der Justiz zu beseitigen, $d$ Riegel, die die Richter einengen, aufzusprengen, das ist - so scheint es mir - st langen Jahren eines der Ziele des »s. m.«. »S. m. «scheint aber doch etwas zu zöger die Dinge beim Namen zu nennen und laut und öffentlich zu proklamieren, daß für die Unabhängigkeit der Justiz kämpft.

\section{Theoretische Unzulänglichkeiten}

Diese Zurückhaltung mag sich damit erklären - es ist schon angesprochen worden daß die Konservativen in bestimmter Weise den Begriff der Unabhängigkeit $d_{1}$

I Bezogen auf Italien und Frankreich; für die Bundesrepublik siehe $§ 2$ Ie GVG. 
Justiz besetzt haben; aber dieser einseitige Gebrauch war seinerseits (nur) möglich auf der Grundlage zweier höchst fragwürdiger theoretischer Voraussetzungen.

Die erste besteht darin, unter der Unabhängigkeit nur die äußere Unabhängigkeit zu verstehen, d. h. die Unabhängigkeit der Justiz im Verhältnis zur Exekutive, ohne sich zur gleichen Zeit mit der inneren Unabhängigkeit zu befassen, nämlich derjenigen jedes einzelnen Richters im Verhältnis zur Hierarchie in der Justiz und zur Richterschaft insgesamt. Das Resultat einer Unabhängigkeit nur nach außen ist eine Justiz, die intern die Hierarchie verinnerlicht hat und deren Spitzen niemand gegenüber verantwortlich sind; eine Justiz, die nicht pluralistisch ist, die eine große Machtfülle in den Händen der Chefpräsidenten konzentriert und vor allem dann und dort vö]lig auf Distanz geht, wenn und wo ein grundlegender Wechsel in der politischen Verantwortlichkeit vor sich geht: Das war der Fall für die chilenische Richterschaft unter Allende ${ }^{2}$.

Eine so geartete Richterschaft ist eine Bastion des Konservatismus, denn sie schließt den Richter in eine bürokratische Struktur ein, schottet ihn ab gegen die ihn umgebende Gesellschaft und alle dort kursierenden Ideen und liefert ihn der Vereinnahmung durch seine Justizoberen aus, ohne daß er eine Chance hätte, sich dagegen zu wehren. Darüber hinaus bringt es die starke Konzentration unkontrollierter Macht, die in der Spitze der Hierarchie stattfindet und die ihrer Natur nach zutiefst undemokratisch ist, mit sich, daß diese dazu neigt, sich von den konservativen Machtzentren angezogen zu fühlen und um diese zu kreisen.

Aus all diesen Gründen sind sich die hellsten Köpfe unter den Politologen darin einig, daß die Unabhängigkeit der Justiz notwendigerweise begleitet sein muß von der Aufteilung der richterlichen Gewalt unter alle Richter in der Weise, daß jeder von ihnen von der gesamten Gewalt einen minimalen Anteil ausübt, die ihr Gegengewicht erhält durch den gleichen Anteil an der richterlichen Gewalt, der jedem seiner Kollegen zugestanden ist, die wie er unabhängig sind. Die Unabhängigkeit insgesamt verweist zurück auf eine Gewalt, die in sich weit gefächert ist, und diese wieder ist nicht vorstellbar ohne innere Unabhängigkeit.

Die andere theoretische Voraussetzung, die eng mit der ersten verbunden ist, besteht darin, die Unabhängigkeit nur als solche im Verhältnis zur Gesellschaft zu verstehen, als Unabhängigkeit im Verhältnis zur öffentlichen Meinung, mehr denn als Unabhängigkeit von der Macht der Apparate; von daher rührt es, daß jegliche Kritik an der Tätigkeit der Justiz als Attentat auf die Unabhängigkeit zurückgewiesen wird.

Diese Position, die die Justiz als der öffentlichen Auseinandersetzung entzogen betrachtet (was ein Zurückbleiben im Verhältnis zu einer lebendigen Demokratie bedeutet) und die sich dabei auf die Unabhängigkeit der Justiz beruft, verkennt, daß im Gegenteil die Justiz notwendigerweise ihr Gegengewicht in einer möglichst umfassenden Kritik und Debatte über die Tätigkeit der Richter finden muß. Um es zusammenzufassen: Die Unabhängigkeit ist Voraussetzung dafür, daß sich Kritik auswirken kann auf die Justiz, Kritik nicht in Form von Pressionen der Exekutivmacht, sondern aus dem Bereich der Kultur und der Ideen. Dieser Zusammenhang zwischen Unabhängigkeit der Justiz und Kritik durch die öffentliche Meinung läßt sich so charakterisieren, daß der Richterberuf die größte Unabhängigkeit gegenüber der Exekutive und die größtmögliche Offenheit gegenüber der öffentlichen Meinung und der Gesellschaft verlangt. Verliert man diesen Zusammenhang aus dem Auge, kann die Unabhängigkeit zum Schutzschild werden, hinter dem sich eine Justiz verbirgt, die stets zu Diensten ist: Haben wir es nicht allzu oft erlebt, daß die

2 Ebenso für die deutsche Richterschaft in der Weimarer Republik. 
Unabhängigkeit der Justiz nur beschworen wurde, um die Diskussion über eine zweifelhafte Justizaffäre zu ersticken? Oder haben wir nicht ebensooft diejenigen, die sich zu Aposteln der richterlichen Unabhängigkeit gegenüber der öffentlichen Meinung machen, sagen hören (dann nämlich, wenn Reformen des Richtergesetzes und der Gerichtsverfassung gefordert wurden, um die Unabhängigkeit zu stärken), richterliche Unabhängigkeit sei nicht eine Frage besserer gesetzlicher Regelungen, sondern eine Frage des Charakters, die Unabhängigkeit müsse nach außen verteidigt werden, weil man doch innerhalb der Richterschaft eine große Familie sei, die wirkliche Bedrohung gehe nicht von der Hierarchie, sondern von der Politisierung aus ...?

\section{Nur eine unabhängige Justiz kann eine demokratische Justiz sein}

Ebenso groß ist die Zahl der Gemeinplätze, die dafür verantwortlich sind, daß im demokratischen Bewußtsein der Begriff und die Tragweite der Unabhängigkeit der Richter verdunkelt worden sind! Dabei handelt es sich um einen institutionellen Wert, der, einmal in richtiger Weise in die Wirklichkeit umgesetzt, fortschrittliche Möglichkeiten zum Vorschein bringt. Und das in mehrerlei Weise.

Zunächst einmal ist eine unabhängige Justiz ein Faktor der Dekonzentration der Staatsmacht. Zu einer Zeit, in der man eine horizontale Machtverteilung durch Stärkung der Regionen anstrebt, mag die Frage angebracht sein, ob man nicht eine Form der Dezentralisation der Staatsgewalt ins Auge fassen sollte, die die Macht in der Gesellschaft aufteilt, denn der Charakter einer breitverteilten Gewalt, den die Justiz in der Folge einer vollen Unabhängigkeit hätte, würde notwendigerweise Aufteilung (und Verankerung) in der Gesellschaft bedeuten, Öffnung gegenüber den verschiedensten Anregungen und Anforderungen, die aus der Bevölkerung kommen.

Aber das ist noch nicht alles. Die institutionell gewährleistete Unabhängigkeit ist ein Faktor, der den Richter verantwortlicher werden läßt: Er kann nicht mehr sein Gewissen beruhigen und sich hinter den Zwängen der Hierarchie und dem Gewicht der Apparate verstecken; er ist frei, also ist er voll und ganz betroffen. Ist der Richter in dieser Weise unabhängig, gewinnt seine Tätigkeit - im weiten Sinne des Wortes kulturelle Bedeutung, Bedeutung für die politische Kultur: Es wird mehr und mehr zu seinem Selbstverständnis gehören, verantwortlich Position zu beziehen gegenüber den unterschiedlichen Lehren, die sich in der Gesellschaft, der Kultur, der Gesetzgebung jeweils gegenüberstehen; seine Stellungnahme fügt sich ein mitten in die Debatten, die das Land bewegen; er zehrt von ihnen und belebt sie zugleich. All das ist notwendig begleitet von einer kulturellen Emanzipation des Richters, die ihn mehr und mehr instand setzt, alle Elemente wirksam werden zu lassen, aus denen sich die Legalität zusammensetzt, einschließlich derjenigen, die den Standpunkt der arbeitenden Klassen und Schichten der Bevölkerung ausdrücken, die allzuoft in der richterlichen Berufspraxis auf der Strecke bleiben.

Allgemeiner gesagt: Die institutionell gesicherte Unabhängigkeit wird dem Richter helfen, die Gewohnheit anzunehmen, nicht nur auf die Stimme der Herrschenden zu hören, sich vielmehr auch den Stimmen von Minderheiten, Randgruppen und Isolierten zu öffnen. Das bedeutet beileibe nicht, daß alles, wofür sich die Richter dann entscheiden werden, progressiv ist, im Gegenteil! Auf nahe Sicht wird sich diese kulturelle Emanzipation kaum in die Wege leiten lassen, ohne daß zunächst das folgende Ergebnis sich zeigt: Wenn die gegenwärtige Justiz konservativ ist, ist es hochwahrscheinlich, daß sie die institutionelle Unabhängigkeit dazu benutzen wird, die Wahl im konservativen Sinne zu treffen. Aber es kommt wenig darauf an, ob die Position, die der einzelne Richter bezieht, fortschrittlich ist oder nicht; selbst wenn 
sich manche als konservativ zu erkennen geben würden, wäre schon die Tatsache,

daß sie sich zu erkennen geben, für sich genommen progressiv; und darauf käme es letzten Endes allein an.

$\mathrm{Da}$ die Justiz ihren autoritären Charakter verliert und nicht mehr im Namen Gottes, des Königs oder irgendeiner esoterischen Rechtstechnik den Menschen etwas auferlegt, sondern ihre Legitimation daraus gewinnt, daß sie sich bewußt und nachvollziehbar anlehnt an diesen oder jenen der unterschiedlichen Standpunkte, die sich in der Gesellschaft gegenüberstehen - darin allein liegt ein unleugbarer Gewinn auf dem Gebiet der Demokratie, der es nicht darum geht, die konservativen Standpunkte auszulöschen, sondern ihnen die Waffen der Vernunft entgegenzusetzen.

Im übrigen wird auf dieser selben kulturellen Ebene die Unabhängigkeit reguliert und vor einem Abgleiten in Willkür geschützt: Wofür sich nämlich tatsächlich der unabhängige Richter entscheidet, er muß sich immer innerhalb der großen Koordinaten halten, die durch die Grundwerte unserer Verfassungen ${ }^{3}$ vorgegeben sind; andernfalls würde er in nicht wieder gutzumachender Weise seine getroffene Entscheidung und seinen Standpunkt diskreditieren. Aber auch von diesen Ausnahmefällen abgesehen, liegt - in einem System unabhängiger Richter - die Überzeugungskraft einer richterlichen Entscheidung in dem Grad der Übereinstimmung mit den verfassungsrechtlichen Grundwerten begründet und der Fähigkeit des Richters, diese Werte zu interpretieren und lebendig werden zu lassen. Dank diesem Bezug auf vorgegebene Leitprinzipien entgeht die richterliche Tätigkeit der Gefahr des Subjektivismus und findet einen plausiblen äußeren Bezugsrahmen. Gerade dieser Rahmen kann im übrigen die Haltung des Richters stärken, der so, gekräftigt durch seine Unabhängigkeit, dem Gift der Repression widersteht, das in Zeiten der Krise von der Mehrheit des Sozialkörpers abgesondert wird.

Die Unabhängigkeit der Justiz erweist sich so nicht nur als Abwehrschranke gegenüber der Exekutive, sondern auch als Hilfsmittel, wenn es darum geht, die Rationalität in Erinnerung zu rufen, dann nämlich, wenn die öffentliche Meinung den gerade gängigen emotionalen Versuchungen des "gesunden Volksempfindens« erliegt.

Die Unabbängigkeit als notwendige institutionelle Form für gesellschaftlich-politischen Wandel

Die Justiz, die ich beschrieben habe, ist gewiß nicht die Justiz von heute. Aber man wird sich fragen müssen, (I.) ob eine solche Justiz anzustreben ist und (2.) ob sie möglich ist.

Wenn man beide Fragen mit Ja beantwortet, erweist sich die Unabhängigkeit der Justiz für die Demokratie als strategischer institutioneller Wert, und ihre volle Verwirklichung stellt sich als ein Ziel dar, wenn man gesellschaftlich-politische Veränderung im Auge hat. Was mich betrifft, so ist in mir die Überzeugung gereift, daß in einem industrialisierten Land mit starker liberal-demokratischer Tradition die Unabhängigkeit der Justiz nicht nur mit gesellschaftlich-politischem Wandel zu vereinbaren ist, sondern daß sie sich nur im Zusammenhang mit einem solchen Wandel vollständig verwirklichen kann. In der Tat ist nicht zufällig in unseren Ländern die Unabhängigkeit der Justiz unvollendet: Eine wirkliche Unabhängigkeit erfordert einen hohen Grad von Demokratie (d. h. Demokratisierung aller gesellschaftlichen Bereiche), eine Hegemonie-Fähigkeit und eine breite Basis des Konsens, was alles wahrscheinlich die herrschenden Klassen niemals gehabt haben.

3 Dieser Begriff scheint mir die bekannte Gefahr der (je nach Belieben rechten oder linken) »Grundgesetztheologie " in sich zu bergen; "die Grundrechte" oder "unsere Verfassungen " schiene mir bei allem Streit, der auch dann noch entbrennen könnte, besser. Das wäre - hier und mit Senese u. a. - zu diskutieren. 
Andererseits bietet sich die Unabhängigkeit der Justiz heute als eine dieser »verfeinerten institutionellen Lösungen « an, die der gesellschaftlich-politische Wandel braucht. Sie ist daher nicht nur mit dem Wandel vereinbar, sondern notwendig für ihn. Ein Prozeß der Veränderung in unseren Gesellschaften erscheint mir tatsächlich möglich, jedoch nur unter der Bedingung, daß er begleitet ist von einer Ausweitung der Freiheiten, von einer gesteigerten Präsenz der Massen im politischen Leben des Landes, sowie unter der weiteren Bedingung, daß die Projekte, die abwechselnd ausgearbeitet werden, sich ständig der Kritik der Bürger stellen. All das verlangt Institutionen, die Freiheiten, Grundrechte, Diskussion und Kritik garantieren. Eine unabhängige Justiz, so wie ich sie oben beschrieben habe, gehört sicher zu diesen Institutionen.

Man kann sich natürlich fragen, welche Legitimation ein aufgrund Ausschreibung und Auswahlwettbewerb eingestellter Berufsrichter dafür hat, das Instrument des Zweifels und des Infragestellens gegenüber einem Projekt oder einer Maßnahme zu sein, die sich auf einen Prozeß stützt, der von einer Mehrheitsentscheidung des Volkswillens ausgelöst ist; und man riskierte, sich über diese Frage festzubeißen, wenn man sich nicht in Erinnerung riefe, daß der radikale Zweifel eine Notwendigkeit des Prozesses der Veränderung ist: Der Prozeß der Veränderung hat keine andere Möglichkeit des Gelingens, als wenn er sich unablässig mit der Kritik und den Einwänden auseinandersetzt, die er hervorruft. Der Richter, der dem Zweifel die Möglichkeit gibt, sich zu entfalten, indem er ihm den ihm zukommenden Platz einräumt innerhalb der Grundwerte des Sozialpaktes, ist Ausdruck einer institutionellen Form, die für den Wandel angemessen und notwendig ist. Diese Form mag dazu benutzt werden können, den Prozeß zu bremsen oder gar zu verhindern; aber dies nur dann, wenn die Kräfte der Veränderung nicht zu der Fähigkeit gelangten, auf dem Gebiet der Kultur und der Ideen eine starke Hegemonie zu entfalten, denn eine wirklich unabhängige Dritte Gewalt kann nur ihren Weg finden vermittelt durch die Kultur, die sie umgibt.

Und deshalb setzt eine volle Unabhängigkeit der Justiz eine Regierungsmacht voraus, die auf gesellschaftlich-politischen Wandel gerichtet ist und die sich der Kraft ihrer Idee gewiß ist.

Salvatore Senese

\section{Anmerkung 1:}

Anders als Hans-Ernst Böttcher halte ich es nicht für erstaunlich, daß dieser Text auch in der justice des syndicat de la magistrature zunächst »nicht und dann nur stark gekürzt" abgedruckt worden ist. Salvatore Senese läßt zu viele Fragen offen.

Ist es wirklich so - so ist zu fragen -, "daß die Justiz ihren autoritären Charakter verliert" und eine neue "Legitimation daraus gewinnt, daß sie sich bewußt und nachvollziehbar anlehnt an diesen oder jenen der unterschiedlichen Standpunkte, die sich in der Gesellschaft gegenüberstehen «?

Um jedes Mißverständnis auszuschließen: Die Veröffentlichung einer »dissenting opinion « in den Entscheidungen des Bundesverfassungsgerichts ist gegenüber der unnahbaren Justiz (im Namen Gottes, des Königs oder des Volkes) zunächst ein großer Fortschritt. Selbst im Rahmen eines institutionell inhaltlich begrenzten Pluralismus kann die Veröffentlichung einer abweichenden Meinung deutlich machen, daß Rechtsprechung von Menschen gemacht wird mit unterschiedlichen politischen Positionen und Vorurteilen im Rahmen politisch-gesellschaftlicher Widersprüche.

Senese sagt, es wäre ein Fortschritt, »wenn sich manche als konservativ zu erkennen 
geben würden«. Er übersieht, daß die Kunst gerade der Mehrheit der konservativ eingestellten Richter zumindest in Deutschland (aber wohl nicht nur in Deutschland) darin besteht, ihre politischen Positionen durch allgemein »anerkannte« oder "gültige " Begriffe zu verschleiern. Diese Richter wissen, unter der Maske des Allgemeinen gedeiht ihr klassenspezifisches Eigeninteresse. Der "linke» politische Richter, der "sich bewußt und nachvollziehbar anlehnt" an eine politisch-gesellschaftliche Position, gerät dagegen ins Abseits; er vertritt offen politische, d. h. nicht rechtliche Positionen. Ein solcher Richter kann als befangen abgelehnt werden. Da niemand den konservativen Richter zwingen kann, seine eigene politische Position auch nur anzudeuten (und es Erfahrung voraussetzt, die politische Position in allgemeinen Begriffen zu erkennen), ist es m. E. - zumindest in der Bundesrepublik für einen Richter im Rahmen einer Rechtsentscheidung nicht ratsam, »sich bewußt und nachvollziehbar« an eine politisch-gesellschaftliche Position anzulehnen.

Etwas anderes ist es, wenn wir von Richtern verlangen, "Positionen zu beziehen gegenüber den unterschiedlichen Lehren, die sich in der Gesellschaft, der Kultur, der Gesetzgebung jeweils gegenüberstehen". Doch das kann nicht dadurch geschehen und das meint auch Senese nicht -, daß der Richter schlicht optiert für den »Standpunkt der arbeitenden Klassen und Schichten «, für die "Stimmen von Minderheiten, Randgruppen und Isolierten «. Senese reduziert das Problem allerdings auf die Formel, die »Emanzipation des Richters « setze diesen instand, »alle Elemente wirksam werden zu lassen, aus denen sich Legalität zusammensetzt«.

Was aber heißt das? Hätte Senese nicht gerade an dieser Stelle mehr ins Detail gehen müssen? Gegen den ausdrücklichen Wortlaut eines Gesetzes haben auf Grund der politisch-gesellschaftlichen Machtverhältnisse in der Bundesrepublik nur konservative Richter Entscheidungen getroffen. Wer sich nicht auf solche außerrechtliche Kräfte stützen kann (oder will), wird ganz im Sinne der klassischen Interessenjurisprudenz zunächst darlegen müssen, daß die gesetzliche Regelung eine Lücke läßt; er wird dann fragen, welche Interessenbewertung liegt der gesetzlichen Regelung zu Grunde? Im Rahmen einer Übertragung der Interessensbewertung des Gesetzes auf die Interessen, die im Rahmen der Ausfüllung der Gesetzeslücke zu berücksichtigen sind, kann der Richter »Elemente wirksam werden lassen «, "aus denen sich Legalität zusammensetzt«. Wenn Senese das meint, warum sagt er es nicht?

Jürgen Seifert

\section{Anmerkung 2:}

Senese fordert nicht den "politischen Richter « in dem platten Sinne, daß dieser "seine " Politik an die Stelle des Gesetzesauftrages setze. Das wäre gerade die Position der von Senese kritisierten Justiz autoritären Charakters: Diese legt das Gesetz nicht aus, sondern legt ihm etwas unter, gibt das ganze noch als die einzig richtige Interpretation aus und schilt die Anhänger anderer Positionen, sie hielten sich nicht an Gesetz und Recht, seien also gar Verfassungsfeinde (Art. 20 Abs. 3 GG). Ganz anders Senese: Die Justiz ist ein plurales Gebilde. "Das Gericht «, »die Justiz« sind im einzelnen und insgesamt Frauen und Männer, Personen mit langen und kurzen Haaren, Christen und Ungläubige, Kriegsdienstverweigerer und Militär-Reservisten, Jüngere und Ältere, Menschen unterschiedlicher Herkunft, Gewerkschafter und Standesvertreter, politisch unterschiedlich denkende und handelnde Individuen. Sie alle haben das Gesetz anzuwenden - das ist ihr Auftrag, nichts sonst. Nichts ist dabei selbstverständlicher, als daß sie jede(r) bei ihrem Bemühen, das Gesetz authentisch anzuwenden und auszulegen, ihre Anschauungen einfließen lassen - je bewußter und je offener, je mehr und klarer in Auseinandersetzungen mit anderen Stand- 
punkten, desto besser. All dies ist im übrigen in der Methodendiskussion mittlerweile Gemeingut. Es kann nicht davon die Rede sein, daß Senese der Meinung das Wort redet, Staatsanwälte und Richter sollten ihre politische Option an die Stelle der Normen setzen. Erstaunlich ist, daß man dies dennoch aus dem Text herausgelesen hat. Seneses Beitrag sollte diskutiert werden.

Hans-Ernst Böttcher

Kolloquium des Instituts für Marxistische Studien und Forschungen (IMSF), Frankfurt/M.

„Marxismus, Staat und Recht heute. Theoretische und aktuell-politische Aspekte"

Samstag, 19. Mai 1984, $10.00 \mathrm{Uhr}$, im Kleinen Saal des Volksbildungsheims Frankfurt/M., Eschenheimer Turm/Oeder Weg.

Vorträge und Diskussion - Es referieren:

Prof. Dr. Josef Schleifstein: „Staatstheorie bei Marx, Engels und Lenin«

Prof. Dr. Heinz Wagner: "Marxistische Rechtstheorie in ihrer aktuellen Bedeutung"

Claus Skrobanek-Leutner: »Aktuelle Entwicklungstendenzen des Staatsapparats im staatsmonopolistischen Kapitalismus der BRD unter der Rechtsregierung «

Dr. Martin Kutscha: „Sozialstaatsverheißung und innerstaatliche Feinderklärung - der Wandel der Verfassungspositionen«

Diskussionsbeiträge sind u. a. angekündigt von Prof. Dr. W. Abendroth, Dr. Schneider, Dr. E. Siemantel.

Teilnehmerbeitrag (einschl. Mittagessen) 10,- DM.

Anmeldung bei: IMSF, Liebigstr. 6, 6000 Frankfurt/M. 